\title{
Performance-aware Replication of Distributed Pre-recorded IPTV Content
}

\author{
Seung-Bum Lee, Student Member, IEEE, Gabriel-Miro Muntean, Member, IEEE, \\ and Alan F. Smeaton, Member, IEEE,
}

\begin{abstract}
Video recording in IPTV systems is a promising service that provides time-shifted services in relation to storing TV content closer to user devices such as set-top boxes. Existing approaches do not support collaboration between nodes which have correlated contents, a fact that can affect the performance of the overall system. To make this service more interactive and proactive, this paper presents the architecture using the Smart Personal Information Network (Smart PIN) as a novel performance-based content sharing network for IPTV content which uses a user-centric utility-based Multimedia Data Replication Scheme (MDRS). This allows the exchange of data based on both network performance and user interest in exchanged multimedia content in order to achieve efficient content sharing. The proposed solution is evaluated through extensive simulations and results show much improved behaviour in comparison with two other existing general purpose data replication schemes.
\end{abstract}

Index Terms-IPTV, Data replication, Content management, Peer-to-peer.

\section{INTRODUCTION}

Internet Protocol Television (IPTV) involves the provision of TV-related services via IP-based networks and among the most important services are IP multicast-based TV broadcasting and interactive Video-on-Demand (VoD). These IP-based networks support high speed Internet as a default and as part of the client-server paradigm, an increasing portion of file sharing and live streaming is based on peer-to-peer (P2P) solution [1], [2], [3]. In the context of IPTV, although P2P-based live streaming has limitations in relation to its startup delay and, channel zapping delay [4] it also provides significant benefits to viewers in terms of quality.

Lately, a third important IPTV service has been emerging: recording live TV programs and distributing pre-recorded multimedia content [5], [6]. This service is used in conjunction with the time-shift or caching-based solutions [7] and enables users to watch TV programs cached on the IPTV servers or in the network or saved on set-top boxes (STBs). However there are many issues related to the efficient usage of the delivery network's capacity and storage space. This is mainly because the different user-located devices do not cooperate although most of the time they contain content which is temporally, spatially and logically correlated. Even when caches are used,

S.-B. Lee and G.-M. Muntean are with School of Electronic Engineering, Dublin City University, Glasnevin, Dublin 9, Ireland. Email: \{sblee, munteang\}@eeng.dcu.ie, Website: http://www.eeng.dcu.ie/ sblee and http://www.eeng.dcu.ie/ munteang.

A.F. Smeaton is with the CLARITY CSET, Dublin City University, Glasnevin, Dublin 9. Ireland.

Manuscript received May 7, 2008. the situation does not improve as these are passive and no interaction between the service users takes place. By enabling data sharing between user devices, enhanced services could be provided. For example, users could play past programs although they did not record them if other users have either watched or recorded them. Also if users missed the start of a program of interest, they could have access to the missed content from other users' devices via the IP network. These enhancements could make existing time-shifted services more interactive and proactive. In this context multi-sender distributed streaming was proposed which enables nodes to receive multimedia data from multiple sources [8], [9], [10], [11], [12]. This requires storing IPTV recorded content in distributed sources and sharing it.

In order to support this IPTV service at a high quality, this paper proposes a novel architecture and scheme for performance-oriented sharing and replication of multimedia content between different distributed users in an IPTV system. A Smart Personal Information Network (Smart PIN) is introduced as a performance-based personal information network which uses a user-centric utility-based Multimedia Data Replication Scheme (MDRS) to exchange data automatically based on both network performance and user interest in the exchanged multimedia content.

This paper is structured as follows. In the next section, IPTV related works are presented, while section three presents the newly proposed Smart PIN and MDRS - the novel usercentric multimedia data replication scheme. Simulation setup, scenarios and results of tests which compare the proposed solution with existing schemes are shown in section four. Section five discuses and analyses the test results obtained. Finally, conclusions and future work directions are presented in the last section.

\section{BACKGROUND AND RELATED WORK}

\section{A. IPTV Standardisation}

Worldwide, a number of operators and companies are involved in IPTV standardisation through the Alliance for Telecommunications Industry Solutions (ATIS), International Telecommunication Union Telecommunication Standardization Sector (ITU-T), Digital Video Broadcasting (DVB), etc. [6]. These propose standards which focus not only on the usual service-related aspects such as portability, scalability, interoperability, performance and accounting, but also on content protection and architectural elements. There are various architectural models of an IPTV distribution network, however, 


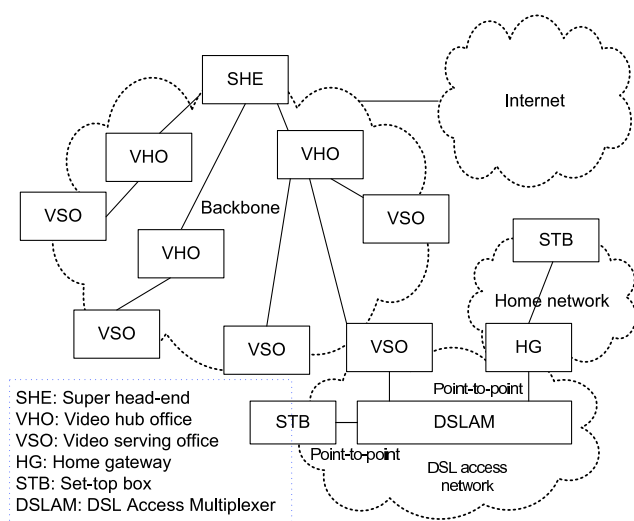

Fig. 1. A typical IPTV network architecture with DSL access network

the architecture presented in Fig. 1 is commonly accepted as a typical one [13], [14], [15], [16] .

Fig. 1 illustrates how the IPTV backbone network includes the super head end (SHE) and a number of video hub offices (VHO). Both of these support encoding and packetising of broadcast TV channels and transmit through an IP network, though SHE usually covers global video content and VHO handles local video content. Both of these include VoD servers which store movies, cache broadcasted shows, etc. A VHO feeds its content to a number of video service offices (VSO) located in the backbone network of the service provider. Finally, VSOs, which are attached to access networks such as DSL, cable, fibre, wireless, etc, distribute content to users' equipment which could be STBs, modems and/or homegateways (HG).

High speed Internet service in IPTV is also provided through a backbone network in similar fashion to conventional broadband services. Since most IPTV services try to deliver multimedia data, the backbone network is usually highly loaded. Content Distribution/Delivery Networks (CDN) are often used in order to mitigate this problem [7]. However, many Internet services are multimedia-based and data travels through the backbone network, putting additional pressure on it. In this context, a solution which increases the usage of access networks instead of the backbone network would be highly beneficial especially if it supports multimedia delivery and thus achieves better end user quality of experience.

\section{B. Multi-sender Distributed Streaming}

Streaming from multiple-sources has relative benefits compared to single-source streaming especially in relation to coping with issues such as varying network conditions during streaming while maintaining high user perceived quality. Each session between a sender and a receiver could be represented as a multiple communication channel which shows less variance in terms of network characteristics with the increase in aggregated bandwidth. Under these conditions, overlay multicast and unicast-based multiple-source streaming are adopted for live streaming [2] and VoD-like services [11]. There are two kinds of unicast-based multiple-source streaming approaches in terms of division and assembly of content for delivery: interlaced packet assembly [11] and Multiple-description code (MDC) [8], [9], [10].

Layered encoding [8] and multiple-description coding (MDC) [9], [10] can be used in conjunction with multimedia data delivery from multiple and different sources. In this context the proposed approaches divide multimedia data into multiple streams with different characteristics. The receiver uses some or all of the available streams to acquire better quality, dynamically. P2P Adaptive Layered Streaming (PALS) [8] is a receiver-driven approach based on the adaptive delivery of stored layered-encoded streams from multiple sender peers to a single receiver. It uses its own quality adaptation solution for congestion-controlled playback of layer-encoded video over the Internet. Cooperative Networking (CoopNet) [9] is a content distribution approach based on data caching and storing delivered data for their ulterior usage. MDC enables CoopNet to provide robust service against the disturbances caused by frequent join and withdrawl of clients. Finally, COSMOS [10] is a multimedia distribution system based on a protocol which employs both primary and secondary communication channels of the various devices. COSMOS uses the primary channel for streaming from the server, and the secondary channel for sharing multimedia content among the participating devices. MDC is used to achieve good video quality and channel utilisation enabling content adaptation to the network dynamics. COSMOS includes a mechanism for sharing information on videos with beacon messages and even though it is assumed that the system is based on heterogeneous networks, no consideration of the properties of different channels is made.

There are also different approaches from those based on encoding in multiple-sender-based multimedia streaming in P2P networks. Interlaced packet assembly schemes divide a multimedia data stream into a sequence of multiple packets. The multiple nodes transfer those packets to a receiver and the receiver merges them as a single stream for playout. Nguyen and Zakhor [11] proposed a framework for streaming video from multiple mirror sites simultaneously to a single receiver over the Internet. The scheme is based on a receiver-driven protocol which is targetted to achieve higher throughput, increase tolerance to loss and reduce delay due to network congestion using the rate allocation algorithm (RAA) and packet partition algorithm (PPA). PPA supports interlaced multimedia data delivery from multiple sources for the receiver. VMesh [12] supports interactive Video on Demand (VoD) service in P2P networks based on a Distributed Hash Table (DHT). VMesh divides videos into variable length segments and stores them in distributed peers over the Internet in a manner similar to caching. The system adopts a locality-aware segment location algorithm providing less stress to the server and good quality to the client, and a popularity-based segment storage scheme which improves playback continuity.

\section{Cache vs. Replication}

Data replication and data caching are often considered to have similar properties [17]. However, they show different characteristics depending on the related operations as Fig 2 
illustrates. Data caching schemes usually assume that there are servers which have data to be shared. A server provides specific services such as web and email. Other nodes, which are considered as clients, access the server to get data which is located on the server, and receive the required data. In this scenario, data cache schemes [18], [3] enable nodes to cache information on the data (this could be location data or even actual data) and to use it later. In contrast, data replication schemes [19], [20], [21] assume that there are a number of devices which act as peers and have similar capabilities. Usually, peers share their data with adjacent devices in advance to use it later depending on their own replication scheme.

As a general purpose cache scheme, Yin and Cao [18] proposed a cooperative caching scheme for ad-hoc networks. They assumed that the system has a server which provides all data. Cooperative caching which was originally developed for wired technologies, allows the sharing and coordination of cached data among multiple nodes. In this work, CacheData (caching the data in nodes between source and destination during transfer), CachePath (caching the nearest location of data during data transfer), and HybridCache (heuristic switching algorithm of CacheData and CachePath) were proposed. Comparisons between the proposed scheme and other simple approaches show better performance at query time and cache hit ratio in various conditions.

In the context of general purpose data replication schemes, Cuenca-Acuna et al. [20] proposed the Automated Replication (AR) scheme in order to achieve high availability of data in P2P file systems based on PlanetP [22], a toolkit for mediumscale P2P applications. PlanetP includes three sub-systems: a gossipping module, an index storage system and distributed query processing engine, and a lightweight active DHT. In $\mathrm{AR}$, data fragments are replicated randomly on the peers' free space and the estimated availability for a file and fragment is measured periodically. Tempo [21] involves a proactive method of data replication during idle time of devices. To limit the usage of bandwidth, it introduces a bandwidth budget which defines the maximum data size per unit time. With this user-specified parameter (bandwidth budget), Tempo removes bursty data transfer for data fragments in a reactive way and provides the same level of durability as previous solutions, without fluctuations of the data transfer. The decision on the transfer is also related to the bandwidth budget. A major problem with this approach is that it does not consider user interest in the actual multimedia content.

Multimedia data cache schemes are widely used in multimedia streaming in order to enhance performance through temporary storage, so-called "cache" which store some parts of the streaming data. Furthermore, some approaches use this cached data for its adjacent devices as "cache-and-relay" and "cache-and-prefetching". The cache-and-relay mechanism [3] uses caching memory for recently played content and such data could be transferred to requesting nodes (e.g. relay) and be played. Cache-and-prefetching mechanisms [23], [24] extend the cache-and-relay approaches in terms of putting content in the cache in advance. Prefetched contents are also used in order to benefit in terms of improved quality, while paying more in bandwidth and memory.

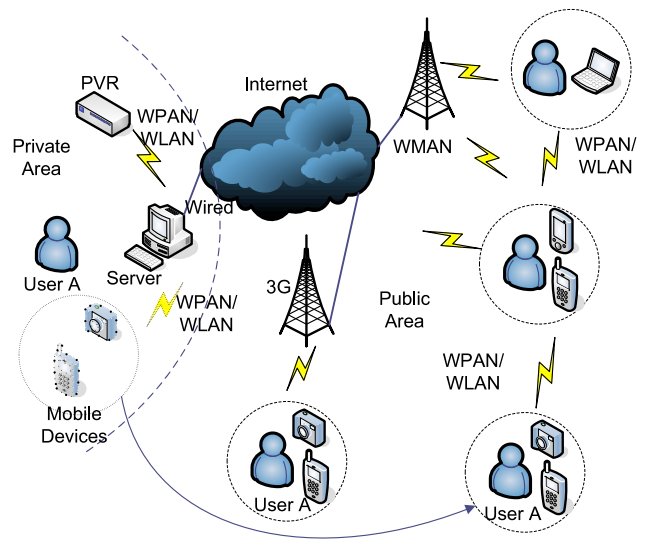

Fig. 3. Overall concept of Smart PIN

As a multimedia data replication scheme, home-to-home online (H2O) [25] is a framework which provides VoD services based on collaborating nodes connected though wired technology. It uses as its replication technique one which considers the worst case expected delay and retrieves a block from one hop away from the current node for the service. Even though there are some simulations with ad hoc networks, H2O does not consider either device or availability as substantial dynamic characteristics of the overall system.

In summary we can say that caching schemes could be the most efficient approaches to managing data delivery since they reuse data which is delivered to a node. However, a cache scheme is basically a reactive approach since the application usually caches data after using it which induces fundamental issue of service availability. For example, if there is not enough data available which makes the service enabled, caching schemes can not guarantee serving that data in the system. On the other hand, replication schemes show lower performance because of initial distribution overhead and intermittent connectivity because of unstable device availability or wireless connections. In spite of that, replication schemes could support specific targets for each scheme, since the approaches are relatively more proactive than caching schemes. Because of this reason, efficient data allocation could be possible with these schemes.

\section{Performance-aware Multimedia Content SHARING AND REPLICATION}

\section{A. Smart PIN}

Smart PIN [26], [27] is a performance and cost-oriented context-aware personal information network which focuses on efficient user access to information located on remotely distributed devices in a heterogeneous network environment. In order to address both information overload, and the heterogeneity of devices and network connectivity, Smart PIN supports a utility function-based data replication scheme as presented in Fig. 3. In order to handle large-size multimedia content, Smart PIN employs data segmentation in fixed length segments (FIX_SEG) and variable length segments (VAR_SEG). Small size data is not segmented and is labelled NO_SEG. 


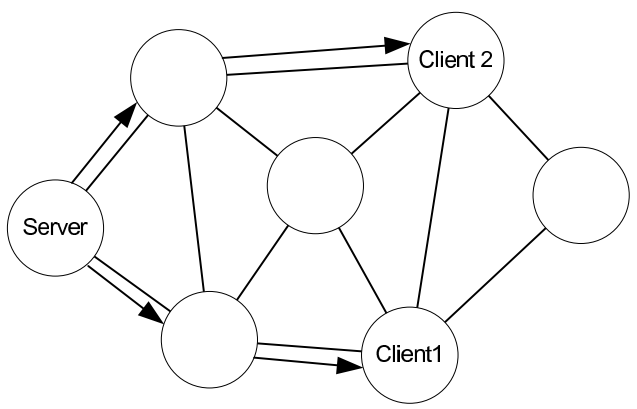

(a) Data cache scheme

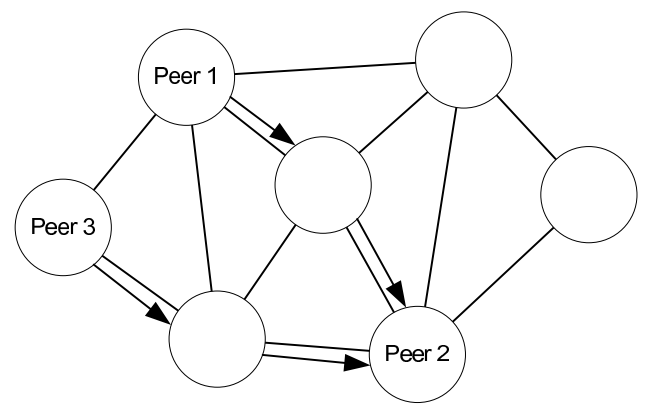

(b) Data replication scheme

Fig. 2. Data cache vs. replication

Smart PIN bases its operation on information gathered from user modelling, device profiling, and metadata associated with each piece of data present in the distributed system. A user model collects information on users' interests and by analysing this information [28], Smart PIN can draw conclusions about a particular user interest in any given piece of data. Device profiles like those built by the User Agent Profile (UAProf) [29] describe the features of the devices from the system and Smart PIN uses information available on the different capabilities of mobile devices to perform data replication and distribution.

\section{B. Utility-based Data Replication in Smart PIN}

Smart PIN bases its functionality on a utility function which includes two main components: the private utility component which reflects the user's individual interest in the content $i$ and the global utility component which expresses the overall utility of the content in relation to its popularity.

The private utility formula includes the content $i$ 's associated benefit $\left(B_{i}\right)$ and its cost $\left(C_{i}\right)$ to the user. In addition, the user interest on the particular data item $i\left(I_{i}\right)$ (e.g. relevance of data to the user) is used to increase or decrease the relative influence of the benefit in comparison with that of the cost. Including normalised values of these metrics, the private utility $\left(P U_{i}\right)$ for item $i$ is computed as in Eq. 1 and as it is normalised, it has values from 0 to 1 . The basic version of Smart PIN described in [27] uses the private utility function only for data replication.

$$
P U_{i}=\frac{1+B_{i} \cdot I_{i}-C_{i}}{2}
$$

The global utility component formula $\left(G U_{i}\right)$ includes apart from the content $i$ 's associated benefit $\left(B_{i}\right)$ and its cost $\left(C_{i}\right)$ - the popularity of the multimedia streaming segment $\left(G_{i}\right)[12]$ as described in Eq. 2.

$$
G U_{i}=\frac{1+B_{i} \cdot G_{i}-C_{i}}{2}
$$

The overall utility function which includes both $P U_{i}$ and $G U_{i}$ is presented in Eq. 3. Different weights are used depending on the data type $(T)$ and its segmentation-related characteristics (i.e. NO_SEG, FIX_SEG and VAR_SEG).

$$
U_{i}=w_{1, T} \cdot P U_{i}+w_{2, T} \cdot G U_{i}
$$

\section{Minimum Data Set Requirement}

Data replication is often used when data is available on distributed devices and their availability differs. The process refers to making copies of data in order to increase their availability to the overall system. In this context, it is not possible to guarantee a successful multimedia streaming process if there is not at least a full set of stable segments of multimedia data available. Therefore to have data availability closest to 1 is desirable.

The availability of a device $\left(P_{j}\right)$ is defined as in Eq. 4. The average data availability is defined in Eq. 5 with the availability of the segment $l$ of the multimedia data item $k$ in a device $j$ as $s_{l, j}^{k}$, device availability $P_{j}$, total number of segments of the multimedia data $k, L$ and total number of devices $J$.

$$
\begin{gathered}
P_{j}=\frac{\text { Device } j \text { available time }}{\text { Total time }} \\
D_{\text {avg }}^{k}=\frac{\sum_{l=1}^{L} \sum_{j=1}^{J}\left(s_{l, j}^{k} \cdot P_{j}\right)}{L \cdot J}
\end{gathered}
$$

It can be shown mathematically that the system availability of the replicated segment $l$ of the multimedia data $k$ across all devices $\left(g_{l}^{k}\right)$ is always greater than or equal to $s_{l, j}^{k}$ as Eq. 6 indicates. By combining Eq. 5 and 6, the relationship from Eq. 7 is derived.

$$
\begin{gathered}
s_{l, j}^{k} \leq \sum_{j=1}^{J} s_{l, j}^{k}=g_{l}^{k} \\
D_{\text {avg }}^{k} \leq \frac{\sum_{l=1}^{L} \sum_{j=1}^{J}\left(g_{l}^{k} \cdot P_{j}\right)}{L \cdot J} \\
=\left(\frac{\sum_{l=1}^{L} g_{l}^{k}}{L}\right) \cdot\left(\frac{\sum_{j=1}^{J} P_{j}}{J}\right)
\end{gathered}
$$

Denoting $P_{a v g}^{J}=\frac{\sum_{j=1}^{J} P_{j}}{J}$ and $G_{a v g}^{L, k}=\frac{\sum_{l=1}^{L} g_{l}^{k}}{L}$, Eq. 7 is simplified as Eq. 8.

$$
D_{\text {avg }}^{k} \leq G_{\text {avg }}^{L, k} \cdot P_{\text {avg }}^{J}
$$

$G_{\text {avg }}^{L, k}$ represents the average availability of multimedia data $k$ in Smart PIN and is dependent on the number of multimedia segment sets in the system. As data availability cannot exceed 
1 and it is desired that the availability to be as high as possible, Smart PIN aims to find the minimum number of sets of segments from the multimedia data $k$ such as for a given average device availability $P_{a v g}$ to have the relationship as defined in Eq. 9. From equation, $G^{k}$ target is derived as in Eq.10.

$$
\begin{gathered}
1=G^{k} \cdot P_{a v g} \\
G^{k}=\left\lceil\frac{1}{P_{a v g}}\right\rceil
\end{gathered}
$$

If $G^{k}$ exceeds the number of total devices $(J)$, some nodes may include more than one duplicated instance of a segment for specific data. Finally, $G^{k}$ could be defined as Eq. 11 .

$$
G^{k}=\min \left(J,\left\lceil\frac{1}{P_{a v g}}\right\rceil\right)
$$

Smart PIN utilises the minimum value for processing segmented multimedia data separately from the other data pieces in order to sustain the minimum set of multimedia segments among the devices. Smart PIN replicates each segment of the VAR_SEG data in order to have the average availability of multimedia data $k$ reach the target value of $G^{k}$ and therefore provide maximum data availability of data given a certain level of device availability. The transfer of the segments is based on the utility function.

\section{Multimedia Data Replication Scheme}

The dynamic characteristics of wireless networks strongly affect distributed application systems as nodes storing shared data can get out of range and suddenly become unavailable. Data replication is usually the main solution in order to achieve high data availability. In this context, Smart PIN employs a novel Multimedia Data Replication Scheme (MDRS), which is divided into two steps, data selection and data delivery, and using the utility function previously described. During data selection, data is classified into three categories based on two thresholds depending on their utility to the users. An example with high, intermediate and low utility groups is presented in Fig. 4. Data from the high utility group will be replicated into devices along with associated metadata. In order to achieve Smart PIN's performance targets, the content from the other two groups is not replicated. However as a user might want to access information with an intermediate utility value, the metadata will be replicated onto the devices along with information on the actual location of the content, allowing fast ulterior access to data. Smart PIN uses a proactive approach based on the introduced utility function, to control network usage. When the system selects data to be replicated, it also decides on data delivery based on the utility function. Smart PIN calculates the transfer duration with the target bandwidth consumption and schedules data replication accordingly. A more detailed description of this algorithm can be found in [26], [27].

In order to apply this algorithm to the IPTV scenario, the system architecture for IPTV multimedia data sharing

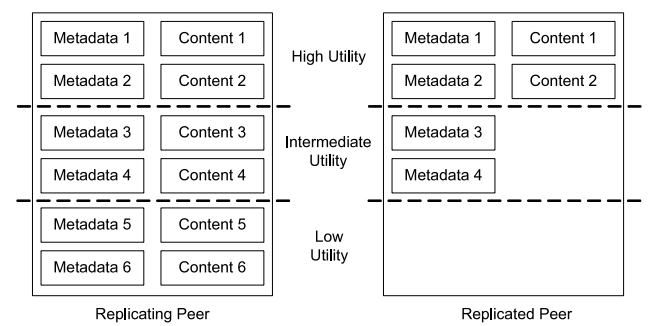

Fig. 4. Data replication using classification in Smart PIN

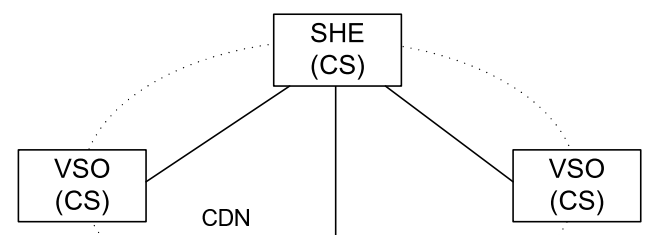
(Server Group)

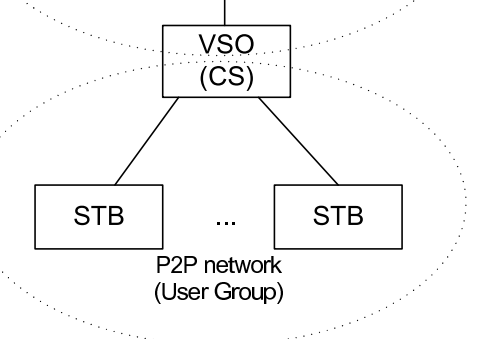

Fig. 5. Proposed architecture for sharing and replication of IPTV multimedia data

and replication is presented in Fig. 5. As shown, apart from VSO, VHE and SHE (Server Group) a Cache Server (CS) is included. These cache and synchronise multimedia content for services such as VoD, recently broadcasted programs through CDN, etc. [7]. Furthermore, VSO works with STBs in the User Group within the IP-based access network. Specifically, VSO and STBs use a P2P network and benefit hashing features such as Distributed Hash Table (DHT). This paper focuses on the operation of the User Group only and variable length segmented (VAR_SEG) multimedia data.

To maximise performance, the User Group keeps the minimum set of multimedia data segments, and data replication should be involved during recording of program $k$. A minimum number of data sets is defined in the previous section and is related to the number of recording nodes (e.g. STBs or PVRs) for a program $k, S_{k}(t)$. It is assumed that program $k$ starts broadcasting from $t_{k, \text { start }}$ and ends at $t_{k, \text { end }}$. Before $t_{k, \text { start }}$, $S_{k}(t)$ denotes the number of nodes which are scheduled for recording of program $k . S_{k}(t)$ represents the number of nodes which are actually recording program $k$ between $t_{k, \text { start }}$ and $t_{k, \text { end }}$. After $t_{k, \text { end }}$, the $S_{k}(t)$ indicates the number of nodes that share the recorded program $k$, and we will now examine 3 possible cases for this.

1) Case 1: $S_{k}(t)=0$ : CS at the level of VSO may cache all broadcasted content if there are no recording nodes. However, there is a limitation of memory to store large amounts of data and because of this, CS just includes recent programs, and old recorded broadcast programs are discarded from storage.

Initially, $S_{k}(t)$ is 0 since there is no node to record the 
TABLE I

PARAMETERS FOR NETWORK MODEL

\begin{tabular}{c|c|c}
\hline Parameter & Downstream & Upstream \\
\hline \hline delayMicros & 5 usecs & 0 usecs \\
\hline capacityPackets & -1 & -1 \\
\hline capacityBytes & 60000 bytes & 60000 bytes \\
\hline lineRateBps & 4 Mbps & 220 kbps \\
\hline
\end{tabular}

program $k$. If $S_{k}(t)$ remains 0 , no recording is performed and no extra action is required.

2) Case 2: $0<S_{k}(t) \leq G^{k}$ : When there are nodes to record a program $k$ (e.g. $S_{k}(t)>0$ ), the minimum multimedia data segment set should be maintained as $G^{k}$. Until $G^{k}$ is met, replication should be performed. CS supports this replication if the program $k$ has already started (e.g. $t>t_{k, \text { start }}$ ). Replication is scheduled based on the Smart PIN algorithm which controls network load.

3) Case 3: $S_{k}(t)>G^{k}$ : This case indicates that the number of recording nodes exceed the minimum data sets required for maximum performance. Therefore no data replication is necessary at this stage. In order to save space, the surplus of data stored could be reduced, but is not the focus of this paper.

\section{Modelling And Simulation}

\section{A. Network Models for Simulation}

The proposed Multimedia Data Replication Scheme (MDRS) has been evaluated via network simulation using the Network Simulator 2 (NS-2) ${ }^{1}$ version 2.30. Residential broadband ${ }^{2}$ NS-2 extension is also used for modelling the wired broadband access network as it supports asymmetric links and enables us to model broadband connectivity such as cable and DSL network. The network parameters for the broadband links between nodes used in the simulation are shown in Table I. Similar to conventional broadband, downstream and upstream links have different bandwidths: 4 Mbps and $220 \mathrm{kbps}$, respectively.

The simulation assumes that the User Group includes 20 STBs and 1 VSO. Therefore, in total 21 simulation nodes are involved. As shown in Fig. 6, two network topologies are considered: a star topology used for a DSL access network and a string topology modelling a cable network [30], [31]. The delivery of a multimedia segment is performed using the TCP protocol which is implemented in NS-2. The used models include Smart PIN-based data replication and support for other protocols or data structures such as DHT and P2P, which are simplified for efficiency purposes.

\section{B. Data Replication Models and Scenarios}

The proposed Multimedia Data Replication Scheme (MDRS) is based on the Smart PIN data replication algorithm described above with multimedia data set availability $G^{k}$ enhancement [26]. To achieve the target minimum data set, each node checks its storage and DHT sequentially. If

\footnotetext{
${ }^{1}$ Network Simulator 2, http://www.isi.edu/nsnam/ns/

${ }^{2}$ Characterizing Residential Broadband Networks, http://broadband.mpisws.mpg.de/residential/
}

TABLE II

PARAMETERS FOR REPLICATION SCHEMES

\begin{tabular}{c|c|c}
\hline & Parameter & Value \\
\hline \hline \multirow{4}{*}{ MDRS } & Transfer utility threshold & 0.75 \\
\cline { 2 - 3 } & Selection utility threshold & 0.75 \\
\cline { 2 - 3 } & Target bandwidth $\left(B_{B W}\right)$ & $1.95 \mathrm{Mbps}$ \\
\cline { 2 - 3 } & Device availability & 0.4 \\
\hline \multirow{3}{*}{ AR } & Replication Interval & 10 secs \\
\cline { 2 - 3 } & Device availability & 0.4 \\
\cline { 2 - 3 } & Target data availability & 0.8 \\
\hline Tempo & Target bandwidth $\left(B_{B W}\right)$ & $1.95 \mathrm{Mbps}$ \\
\hline
\end{tabular}

there is multimedia content which does not have enough data segments, the replicating node picks up a node from the User Group and checks whether it can include specific segments or not. If the replicating node finds such an available node, delivery is scheduled based on the utility function. As this paper focuses on the delivery performance only, all multimedia segments used in the simulation are assumed to have the same utility. Smart PIN and its MDRS are modelled as a NS-2 application with specific parameters which are shown in Table II.

The proposed Smart PIN is compared with two general purpose data replication schemes which are also modelled in NS-2: Autonomous Replication (AR) [20] and Tempo [21]. The implemented AR scheme periodically estimates data segment availability. If the estimation does not reach the target data availability, the replicating node picks up a node at random and transfers the data. Since Tempo considers its network usage, the implemented model picks up a random segment and transfers data based on its network budget (e.g. target bandwidth is indicated in Table II).

In order to put pressure on the delivery network, the data replication simulation uses multimedia sequences from the "Die Hard 1" movie, MPEG-2 encoded at a high quality (4 Mbps). As the replication schemes use variable length segmentation, the multimedia sequences are divided into 102 segments and each segment includes up to 5 Group of Pictures. The average size of the segments is 1.47 Mbytes. The minimum and maximum segment size are 164 kbytes and 3.42 Mbytes, respectively. These values were such set in order to test the case with a wide range of content of variable size.

Test scenarios include recording and data replication as depicted in Fig. 8. The recording operation uses the multimedia sequences provided. When a segment is generated, recording nodes store it in their own storage space. Meanwhile, each data replication scheme is used in turn when the recording starts for comparison. Depending on the scheme used, replicated nodes include portions of data segments from recording nodes. Since a number of recording nodes are involved, the initial number of data segments are set, and each scheme is involved with a different number of recording nodes.

\section{Streaming models and scenarios}

In order to analyse the relationship between streaming and data replication traffic, the simulation adopts streaming approaches based on TFRC and UDP protocols which are commonly used for multimedia streaming [11], [32]. While 


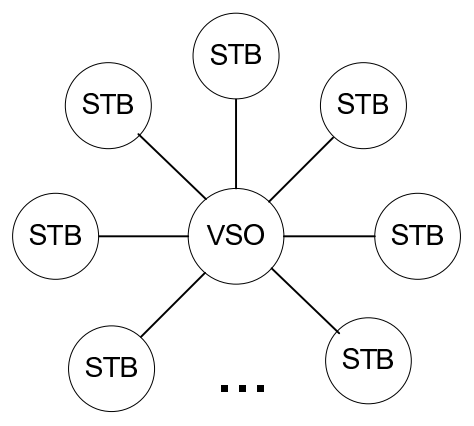

(a) Star topology

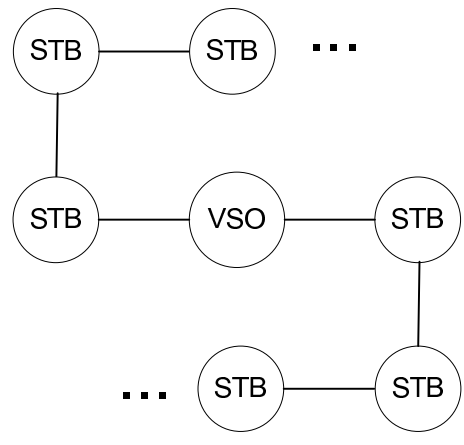

(b) String topology

Fig. 6. Network topology used for simulation (4 Mbps downlink, 220 kbps uplink)

the TFRC traffic is friendly towards TCP transfers, the UDPbased one is not using the bandwidth in a greedy manner. In all scenarios, the receiver uses a buffer to collect data before feeding it to the decoder and schedules the packets for delivery from the senders. The buffer size for each case is set to 1 Mbyte in order to remove time-related constraints such as delay and jitter.

Test scenarios include basic IPTV data replication which supports recording. In addition, a server and a client are used for simulation streaming purposes. The nodes used for streaming are not involved in data replication in order to simplify the simulation and focus on one aspect at a time. Since the current tests consider network characteristics only, the sender stores the whole "Die Hard 1" movie for streaming. The topologies used for testing are presented in Fig 7. Streaming simulation lasts 200 seconds in each test.

\section{Test Results Analysis}

Comparative simulation tests are performed as described in the previous section in order to evaluate the performance of the proposed MRDS. There are two main aspects to be analysed: data and network. In terms of data, the performance reflects if the required data is accessible and what is the overhead for maintaining data to be accessible. In terms of network resource usage for data replication, data loss during delivery is used to measure the performance. Additionally, streaming tests address which combination of data replication and streaming approach shows better performance.

One of the metrics for performance assessment is data availability which is measured with the average online rate of device in the range of communication and ratio of data residing duration over total test time on a specific device as described in Eq. 12 and Eq. 13. Network resource usage is measured by the rate of data received by each device. Loss is measured by the rate of data dropped from all devices.

$$
\begin{gathered}
R_{l, j}=\frac{\text { Data l residing time in device } j}{\text { Total test duration }} \\
D_{\text {avg }}^{k}=\frac{\sum_{l=1}^{L} \sum_{j=1}^{J}\left(s_{l, j}^{k} \cdot P_{j} \cdot R_{l, j}\right)}{L \cdot J}
\end{gathered}
$$

Table III and IV present the results of the comparison between the Multimedia Data Replication Scheme (MDRS) and the other two approaches: AR and Tempo. The assessment is performed in terms of the following metrics: number of data segments (Num.), average data set size (Avg D.S.) and average data availability (Avg D.A.). As assumed and stated in Table II, the device availability is 0.4 . With this value, the minimum data set can be simply calculated by deriving equation Eq 11 . In general, the number of data (Num.) and average data set size (Avg D.S.) expand as the number of recording applications increases. The basic reason for this tendency is simply that the initial number of data replications could be considered as the number of segments multiplied by the number of recording applications. MDRS and AR show that there is no data replication when the number of recording applications is bigger than 3 for AR and 2 for MDRS. However, Tempo does not show saturation for number of data and data sets during the simulation since that considers only the target network usage and any numbers related to data are not used in the algorithm for replication.

In relation to the star topology, the simulation results present interesting values of MRDS when the recording application number is 1 and 2, respectively. Since recording applications provides lower number of segments than the minimum data set (e.g. 3), the nodes which contain the data try to duplicate it into different devices. AR also shows similar results in this topology. However, as it is based on estimated data availability, it tries to replicate more data into other devices than required, unnecessary loading the network and consuming additional storage space. As generally discussed, Tempo shows no saturation and continuously replicates data during the simulation.

Test results, when the string topology is used, show similar characteristics to those when the star topology is employed. MRDS and AR perform no data replication with 2 and 3 recording nodes, respectively. As the number of data in MRDS is targeting the minimum data set (e.g. 3), the replication testing results are very close to this value. On other hand, AR shows higher data replication than MRDS because its estimated data availability is quite low and more data is needed in order to achieve higher value. Tempo also presents similar results to star topology.

Fig. 9 and 10 show the results assessed by the network usage and data loss in each topology. Currently, when the string topology is used, no drop of packets during the simulation are experienced. The assumed string topology includes asyn- 


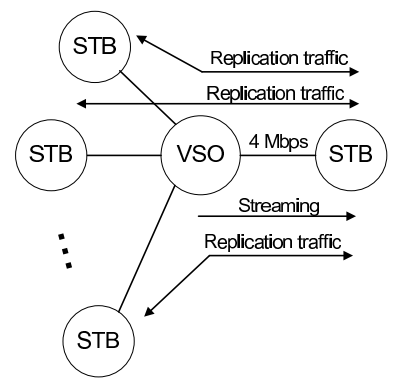

(a) Star topology

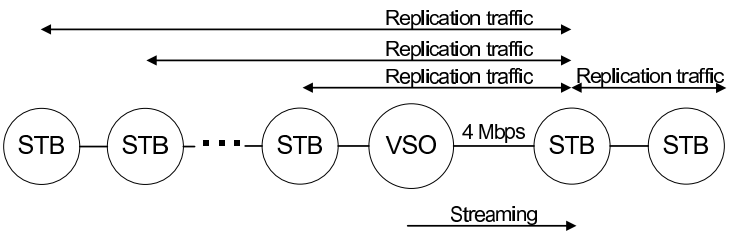

(b) String topology

Fig. 7. Network topology used for streaming simulation (4 Mbps downlink, 220 kbps uplink)

TABLE III

SiMULATION RESULTS STATISTICS IN STAR TOPOLOGY (SIMULATION TIME 2 HOURS)

\begin{tabular}{c|c|c|c|c|c|c|c|c|c}
\hline \multirow{2}{*}{ Recording node(s) } & \multicolumn{3}{|c|}{ MRDS } & \multicolumn{3}{c|}{ AR } & \multicolumn{3}{c}{ Tempo } \\
\cline { 2 - 11 } & Num. & Avg D.S. & Avg. D.A. & Num. & Avg D.S. & Avg. D.A. & Num. & Avg D.S. & Avg. D.A. \\
\hline \hline 1 & 192 & 1.9 & 0.729 & 595 & 5.8 & 0.626 & 842 & 8.25 & 0.428 \\
\hline 2 & 238 & 2.3 & 0.747 & 686 & 6.7 & 0.654 & 1098 & 10.8 & 0.476 \\
\hline 3 & 306 & 3 & 0.980 & 604 & 5.9 & 0.726 & 1386 & 13.6 & 0.541 \\
\hline 4 & 408 & 4 & 0.980 & 408 & 4 & 0.980 & 1543 & 15.1 & 0.557 \\
\hline 5 & 510 & 5 & 0.980 & 510 & 5 & 0.980 & 1631 & 16.0 & 0.592 \\
\hline 6 & 612 & 6 & 0.980 & 612 & 6 & 0.980 & 1771 & 17.4 & 0.629 \\
\hline 7 & 714 & 7 & 0.980 & 714 & 7 & 0.980 & 1856 & 18.2 & 0.662 \\
\hline 10 & 1020 & 10 & 0.980 & 1020 & 10 & 0.980 & 2023 & 19.8 & 0.739 \\
\hline 15 & 1530 & 15 & 0.980 & 1530 & 15 & 0.980 & 2040 & 20 & 0.934 \\
\hline
\end{tabular}

TABLE IV

SIMULATION RESULTS STATISTICS IN STRING TOPOLOGY (SIMULATION TIME 2 HOURS)

\begin{tabular}{c|c|c|c|c|c|c|c|c|c}
\hline \multirow{2}{*}{ Recording node(s) } & \multicolumn{4}{|c|}{ MRDS } & \multicolumn{3}{c|}{ AR } & \multicolumn{3}{c}{ Tempo } \\
\cline { 2 - 11 } & Num. & Avg D.S. & Avg. D.A. & Num. & Avg D.S. & Avg. D.A. & Num. & Avg D.S. & Avg. D.A. \\
\hline \hline 1 & 283 & 2.8 & 0.882 & 1059 & 10.4 & 0.576 & 911 & 8.9 & 0.510 \\
\hline 2 & 331 & 3.2 & 0.943 & 1051 & 10.3 & 0.733 & 1116 & 10.9 & 0.538 \\
\hline 3 & 306 & 3 & 0.980 & 772 & 7.6 & 0.900 & 1393 & 13.7 & 0.596 \\
\hline 4 & 408 & 4 & 0.980 & 408 & 4 & 0.980 & 1496 & 14.7 & 0.608 \\
\hline 5 & 510 & 5 & 0.980 & 510 & 5 & 0.980 & 1707 & 16.7 & 0.652 \\
\hline 6 & 612 & 6 & 0.980 & 612 & 6 & 0.980 & 1494 & 14.6 & 0.682 \\
\hline 7 & 714 & 7 & 0.980 & 714 & 7 & 0.980 & 1500 & 14.7 & 0.729 \\
\hline 10 & 1020 & 10 & 0.980 & 1020 & 10 & 0.980 & 1806 & 17.7 & 0.780 \\
\hline 15 & 1530 & 15 & 0.980 & 1530 & 15 & 0.980 & 1975 & 19.4 & 0.900 \\
\hline
\end{tabular}

chronous connection among the nodes. Specifically, downstream, which has higher bandwidth to compare with the connection in the opposite direction, is mainly used for delivering replication data in the current simulation environment and data sources are mostly located in the nodes utilising this downstream connections. With this reason, data loss for string topology is not presented in this paper. Generally, network usage increases with the number of recording applications. However, MRDS and AR each have saturation points since the amount of replicated data is determined based on their own restrictions as they are monitored in Fig. 10(a) and 10(b).

Fig. 9(a) shows MDRS network usage. As it shows, network usage increases with the number of recording applications. The minimum data set 3 is achieved after the recording application number equals 3 . Because of this, other cases with larger numbers of recording applications show no data replication. Network usage of AR in the string topology is also similar to this situation, increasing until 3 recording applications are reached. After that, there is no data replication, either. In contrast, Tempo shows continuous increases with the number of recording applications. In the string topology, network usage presents a similar tendency.

Fig. 9(a), 9(b) and 9(c) illustrate network load when the star topology was employed for each scheme as it was mentioned. MDRS uses $109 \mathrm{kbps}$ on average when 2 recording nodes are involved, whereas AR and Tempo use 239 kbps and 536 kbps, respectively. These numbers are more than twice that of MDRS. In terms of data loss, MDRS shows 1.7 Kbytes per sec on average with 2 recording nodes. Meanwhile, AR presents 2.8 Kbytes per sec and Tempo shows 2.4 Kbytes per sec on average. These results show much better performance in terms of network load and loss rate when using the proposed MDRS in comparison with when AR and Tempo were used.

Table V and Table VI present throughput and estimated Peak Signal-to-Noise Ratio (PSNR) during multimedia streaming, when the proposed scheme MRDS is compared with two other solutions: AR and Tempo when TFRC and UDP-based streaming is performed. Estimated PSNR is measured based on the throughput during streaming and is expressed in decibels.

In this paper, PSNR is estimated according to the formula 


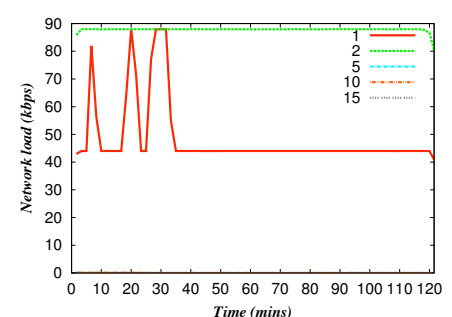

(a) MDRS network load

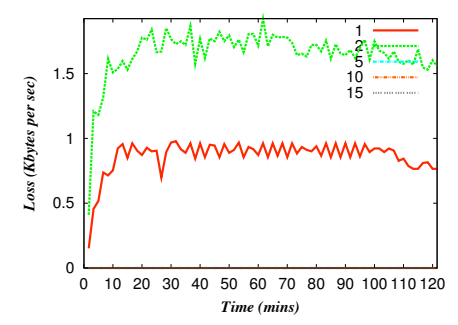

(d) MDRS data loss

Fig. 9. Network load and data loss in star topology

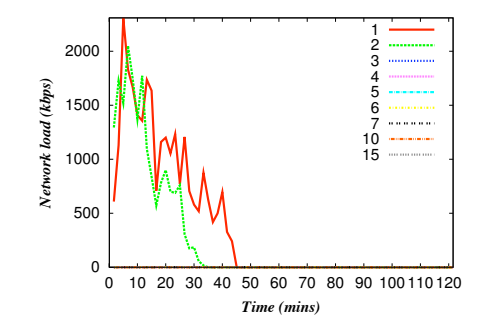

(a) MDRS network load

Fig. 10. Network load in string topology

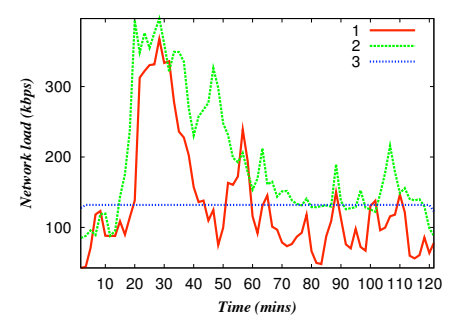

(b) AR network load

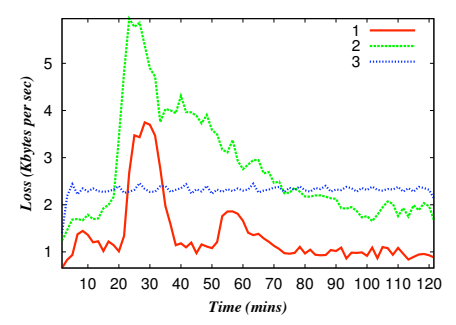

(e) AR data loss

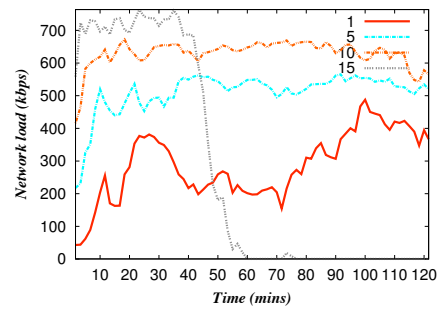

(c) Tempo network load

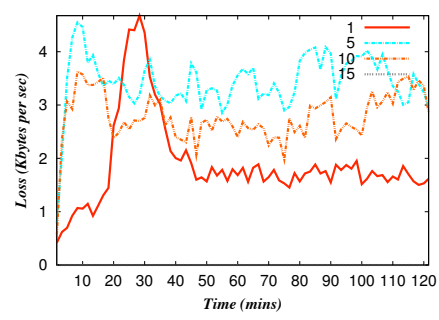

(f) Tempo data loss

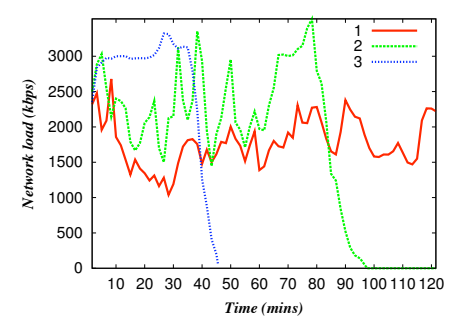

(b) AR network load

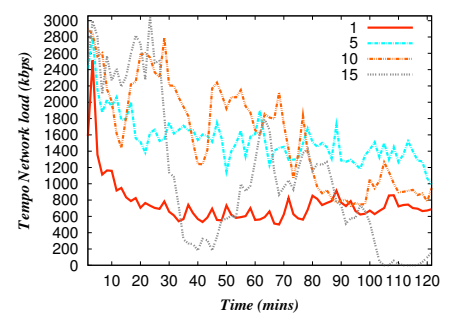

(c) Tempo network load

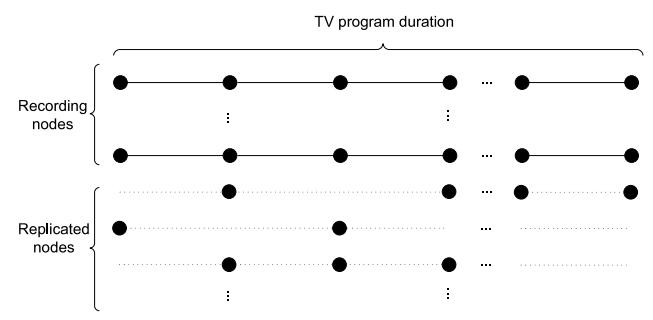

Fig. 8. Simulation scenario for MDRS

presented in Eq. 14 as multimedia quality gets influences from the delivery through communication channel [33]. In Eq. 14, MAX_Bitrate is the average bitrate of the multimedia stream as resulted after the encoding process, EXP_Thr is the average throughput expected to be achieved when adaptively delivering the multimedia stream over the network and $C R T_{-} T h r$ is the actual throughput measured during delivery.

$$
P S N R=20 \cdot \log _{10}\left(\frac{M A X_{\_} \text {Bitrate }}{\sqrt{\left(E X P_{-} T h r-C R T \_T h r\right)^{2}}}\right)
$$

In general, MRDS provides higher throughput and esti- mated PSNR in comparison with the other data replication approaches tested. UDP based streaming shows high and relatively stable throughput since it does not involve any adaptation. TFRC provides adaptation using rate control. In order to do this, the receiver generates feedback and send it to the sender. As the $220 \mathrm{kbps}$ upstream channel is easily filled with data replication traffic, TFRC tries to reduce the multimedia transmission rate affecting user perceived quality.

Table V and Table VI show testing results when star and string topologies are used. When using UDP streaming over the star topology, MDRS shows $8.78 \%$ and $18.88 \%$ improvement against $\mathrm{AR}$ and Tempo in terms of estimated PSNR, respectively. When employing UDP over the string topology, similar PSNR is obtained in all three schemes. A significant benefit of using MDRS is when transmitting using TFRC. In this situation, PSNR is 8 and 4 times higher than AR and Tempo, respectively. When TFRC streaming is applied over the string topology, MDRS shows $30.65 \%$ and $71.14 \%$ improvement against AR and Tempo in terms of estimated PSNR, respectively.

In summary, the proposed MRDS is compared against other general purpose data replication schemes such as AR and Tempo. The tests include scenarios having different number of recording nodes. MRDS shows better performance than the 
TABLE V

RESULTS FOR MULTIMEDIA STREAMING OVER THE STAR TOPOLOGY

\begin{tabular}{l|l|l|l}
\hline $\begin{array}{l}\text { Underlying } \\
\text { protocol }\end{array}$ & $\begin{array}{l}\text { Replication } \\
\text { scheme }\end{array}$ & $\begin{array}{l}\text { Streaming } \\
\text { throughput } \\
\text { (Mbps) }\end{array}$ & $\begin{array}{l}\text { PSNR } \\
(\mathrm{dB})\end{array}$ \\
\hline \hline \multirow{3}{*}{ UDP } & MDRS & 3.98 & 46.98 \\
\cline { 2 - 4 } & AR & 3.97 & 43.19 \\
\cline { 2 - 4 } & Tempo & 3.96 & 39.53 \\
\hline \multirow{3}{*}{ TFRC } & MDRS & 3.94 & 36.86 \\
\cline { 2 - 4 } & AR & 1.43 & 3.86 \\
\cline { 2 - 4 } & Tempo & 2.25 & 7.16 \\
\hline
\end{tabular}

TABLE VI

RESULTS FOR MULTIMEDIA STREAMING OVER THE STRING TOPOLOGY

\begin{tabular}{l|l|l|l}
\hline $\begin{array}{l}\text { Underlying } \\
\text { protocol }\end{array}$ & $\begin{array}{l}\text { Replication } \\
\text { scheme }\end{array}$ & $\begin{array}{l}\text { Streaming } \\
\text { throughput } \\
(\mathrm{Mbps})\end{array}$ & $\begin{array}{l}\text { PSNR } \\
(\mathrm{dB})\end{array}$ \\
\hline \hline \multirow{3}{*}{ UDP } & MDRS & 3.98 & 45.90 \\
\cline { 2 - 4 } & AR & 3.98 & 45.90 \\
\cline { 2 - 4 } & Tempo & 3.98 & 45.90 \\
\hline \multirow{3}{*}{ TFRC } & MDRS & 3.93 & 34.57 \\
\cline { 2 - 4 } & AR & 3.81 & 26.46 \\
\cline { 2 - 4 } & Tempo & 3.61 & 20.20 \\
\hline
\end{tabular}

other general purpose data replication schemes such as AR and Tempo in terms of data availability and network resource usage on different typical topologies for broadband service networks. When streaming is performed with MRDS data replication, better performance in terms of throughput and estimated PSNR in comparison with other two approaches is achieved.

\section{CONCLUSIONS}

In order to enhance the IPTV viewer's experience, the very popular live TV and Video On Demand services are complemented by a third service: recording or time-shifted live TV. In this context, this paper proposes Smart PIN as a novel solution for sharing and replication of multimedia data in IPTV systems. Smart PIN is a performance and costoriented context-aware Personal Information Network which helps IPTV users to exchange multimedia data with other users in order to increase its availability. In order to achieve performance-based replication, Smart PIN uses a novel utilitybased Multimedia Data Replication Scheme (MRDS). The proposed approach is evaluated through simulations and test results show improved performance in terms of a lower number of replicated data segments and decreased network usage in comparison with two other general purpose data replication schemes: Autonomous Replication and Tempo.

The future work will focus on releasing some data from the overloaded nodes and in doing so we can further improve performance. Based on data replication, distributed streaming applications in IPTV systems should also be considered. Finally, balancing background and foreground data delivery will also be addressed in the future.

\section{ACKNOWLEDGEMENT}

The first author gratefully acknowledges the support for this research from Samsung Electronics Co., Ltd.

\section{REFERENCES}

[1] D. A. Tran, K. A. Hua, and T. T. Do, "A Peer-to-Peer Architecture for Media Streaming," IEEE Journal on Selected Areas in Communications, vol. 22, no. 1, p. 2004, Jan. 2004.

[2] X. Zhang, J. Liu, B. Li, and T. Yum, "CoolStreaming/DONet: a datadriven overlay network for peer-to-peer live media streaming," Proceedings of IEEE INFOCOM, Mar 2005.

[3] Y. Cui, B. Li, and K. Nahrstedt, "oStream: asynchronous streaming multicast in application-layer overlay networks," IEEE Journal on Selected Areas in Communications, vol. 22, no. 1, pp. 91-106, Jan. 2004.

[4] X. Hei, Y. Liu, and K. Ross, "IPTV over P2P streaming networks: the mesh-pull approach," IEEE Communications Magazine, vol. 46, no. 2, pp. 86-92, Feb. 2008.

[5] J. Thomson, "IPTV - market, regulatory trends and policy options in Europe," Proceedings of IET IPTV Conference 2007 - Deployment and Service Delivery, pp. 1-32, Dec. 2007.

[6] C.-S. Lee, "IPTV over Next Generation Networks in ITU-T," Proceedings of IEEE/IFIP International Workshop on Broadband Convergence Networks, pp. 1-18, 2007.

[7] T. Wauters, K. Vlaeminck, W. V. de Meerssche, E. Six, and T. V. Caenegem, "IPTV deployment - Trigger for advanced network services!" The Journal of The Communications Network, vol. 5, no. 3, pp. 63-69, Jul.Sep. 2006

[8] R. Rejaie and A. Ortega, "PALS: peer-to-peer adaptive layered streaming," Proceedings of the International Workshop on Network and Operating System Support for Digital Audio and Video, pp. 153-161, 2003.

[9] V. N. Padmanabhan, H. J. Wang, P. A. Chou, and K. Sripanidkulchai, "Distributing streaming media content using cooperative networking," Proceedings of Workshop on Network and Operating System Support for Digital Audio and Video, pp. 177-186, 2002.

[10] M.-F. Leung and S.-H. Chan, "Broadcast-Based Peer-to-Peer Collaborative Video Streaming Among Mobiles," IEEE Transactions on Broadcasting, vol. 53, no. 1, pp. 350-361, 2007.

[11] T. Nguyen and A. Zakhor, "Multiple Sender Distributed Video Streaming," IEEE Transactions on Multimedia, vol. 6, no. 2, pp. 315-326, Apr. 2004

[12] W.-P. Yiu, X. Jin, and S.-H. Chan, "VMesh: Distributed Segment Storage for Peer-to-Peer Interactive Video Streaming," IEEE Journal on Selected Areas in Communications, vol. 25, no. 9, pp. 1717-1731, Dec. 2007.

[13] ATIS Std. ATIS-0800007, "IPTV High Level Architecture," 2007.

[14] C. Luo, J. Sun, and H. Xiong, "Monitoring and Troubleshooting in Operational IP-TV System," IEEE Transactions on Broadcasting, vol. 53, no. 3, pp. 711-718, Sep. 2007.

[15] S. Han, S. Lisle, and G. Nehib, "IPTV transport architecture alternatives and economic considerations," IEEE Communications Magazine, vol. 46, no. 2, pp. 70-77, Feb. 2008.

[16] N. Degrande, K. Laevens, D. Vleeschauwer, and R. Sharpe, "Increasing the user perceived quality for IPTV services," IEEE Communications Magazine, vol. 46, no. 2, pp. 94-100, Feb. 2008.

[17] B.-G. Chun, K. Chaudhuri, H. Wee, M. Barreno, C. H. Papadimitriou, and J. Kubiatowicz, "Selfish caching in distributed systems: a gametheoretic analysis," Proceedings of the twenty-third annual ACM symposium on Principles of distributed computing, pp. 21 - 30, Jul. 2004.

[18] L. Yin and G. Cao, "Supporting Co-operative Caching in Ad Hoc Networks," Proceedings of the IEEE INFOCOM Conference, Mar. 2004.

[19] J. Kubiatowicz, D. Bindel, Y. Chen, S. Czerwinski, P. Eaton, D. Geels, R. Gummadi, S. Rhea, H. Weatherspoon, C. Wells, and B. Zhao, "OceanStore: an architecture for globalscale persistent storage," Proceedings of the ninth international conference on Architectural support for programming languages and operating systems (ASPLOS-IX), pp. 190-201, 2000.

[20] F. M. Cuenca-Acuna, R. P. Martin, and T. D. Nguyen, "Autonomous Replication for High Availability in Unstructured P2P Systems," Proceedings of the 22nd IEEE Symposium on Reliable Distributed Systems (SRDS-22), pp. 99-109, Oct. 2003.

[21] E. Sit, A. Haeberlen, F. Dabek, B. Chun, H. Weatherspoon, R. Morris, M. Kaashoek, and J. Kubiatowicz, "Proactive replication for data durability," Proceedings of the 5th International Workshop on Peer-to-Peer Systems (IPTPS), Feb. 2006.

[22] F. M. Cuenca-Acuna, C. Peery, R. P. Martin, and T. D. Nguyen, "PlanetP: Using Gossiping to Build Content Addressable Peer-to-Peer Information Sharing Communities," Proceedings of the International Symposium on High Performance Distributed Computing (HPDC), pp. 236-246, Jun. 2003. 
[23] A. Sharma, A. Bestavros, and I. Matta, "dPAM: a distributed prefetching protocol for scalable asynchronous multicast in $\mathrm{P} 2 \mathrm{P}$ systems," Proceedings of IEEE Annual Joint Conference of the IEEE Computer and Communications Societies (INFOCOM), vol. 2, pp. 1139-1150, Mar. 2005.

[24] C. Zheng, G. Shen, and S. Li, "Distributed prefetching scheme for random seek support in peer-to-peer streaming applications," Proceedings of ACM workshop on Advances in peer-to-peer multimedia streaming, Nov. 2005.

[25] S. Ghandeharizadeh, B. Krishnamachari, and S. Song, "Placement of continuous media in wireless peer-to-peer networks," IEEE Transactions on Multimedia, vol. 6, no. 2, pp. 335-342, Apr. 2004.

[26] S. B. Lee, G. M. Muntean, and A. F. Smeaton, "Smart PIN: Utilitybased Replication and Delivery of Multimedia Content to Mobile Users in Wireless Networks," IEEE International Symposium on Broadband Multimedia Systems and Broadcasting 2008, Mar. 2008.

[27] — , "User-centric Utility-based Data Replication in Heterogeneous Networks," ICC 2008 Workshop for Digital Television and Mobile Multimedia Broadcasting, May. 2008.

[28] A. Kobsa, "Generic User Modeling Systems," User Modeling and UserAdapted Interaction, vol. 11, no. 1-2, pp. 49-63, Mar. 2001.

[29] "WAG UAProf: Wireless Application Protocol," Open Mobile Alliance (OMA) Standard, Oct. 2001., http://www.openmobilealliance.org/tech/affiliates/wap/wap-248-uaprof20011020-a.pdf.

[30] M. Dischinger, A. Haeberlen, K. P. Gummadi, and S. Saroiu, "Characterizing Residential Broadband Networks," In Proceedings of the Internet Measurement Conference, Oct. 2007.

[31] D. boong Lee, H. Joo, and H. Song, "An Effective Channel Control Algorithm for Integrated IPTV Services Over DOCSIS CATV Networks," IEEE Transactions on Broadcasting, vol. 53, no. 4, pp. 789-796, Dec. 2007.

[32] M. Hefeeda, A. Habib, B. Botev, D. Xu, and B. Bhargava, "PROMISE: Peer-to-peer Media Streaming using CollectCast," Proceedings of ACM Multimedia, Nov. 2003.

[33] N. Thomos, N. Boulgouris, and M. Strintzis, "Optimized transmission of JPEG2000 streams over wireless channels," IEEE Transactions on Image Processing, vol. 15, no. 1, pp. 54-67, Jan. 2006.

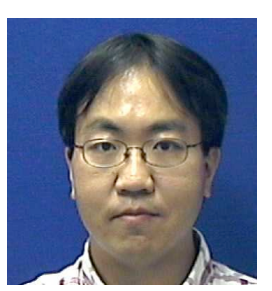

Seung-Bum Lee received the B.S. and M.S. degree in Electronic Engineering from Sogang University, Korea, in 1996 and 1998 respectively. He served as Senior Engineer in Mobile Communication Division, Telecommunication Network Business, Samsung Electronics Co., Ltd until 2006. Currently he is working towards the Ph.D. degree in Electronic Engineering with Performance Engineering Laboratory at Dublin City University, Ireland. Mr. Lee's current research interests include performance issues of data dissemination and replication, and cross-layer issues of service composition management with various devices in heterogeneous network environments. He is a student member of IEEE.

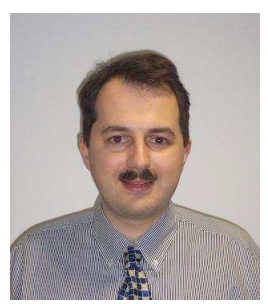

Gabriel-Miro Muntean is a Lecturer with the School of Electronic Engineering, Dublin City University, Ireland, where he obtained his Ph.D. degree in 2003 for research in quality-oriented adaptive multimedia streaming over wired networks. He was awarded the B.Eng. and M.Sc. degrees in Software Engineering from the Computer Science Department, "Politehnica" University of Timisoara, Romania in 1996 and 1997 respectively. Dr. Muntean is a Co-director of the Performance Engineering Laboratory - Ireland research group. His research interests include QoS and performance issues of adaptive multimedia streaming, and personalised eLearning over wired and wireless networks and with various devices. Dr. Muntean has published over 90 papers in prestigious international conferences and journals, has authored a book and five book chapters and has edited two other books. Dr. Muntean is an Associate Editor of the IEEE Transactions on Broadcasting and reviewer for other important international journals, conferences and funding agencies. He is a member of IEEE, Network Inovation Centre and Research Institute for Networks and Communications Engineering, Ireland.

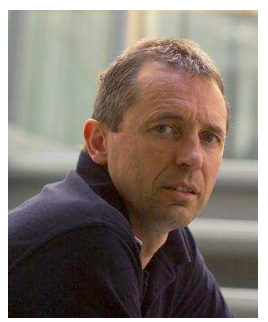

Alan F. Smeaton is a Professor of Computing in the School of Computing at Dublin City University, Ireland. He obtained the B.Sc., M.Sc. and PhD degrees in Computer Science from University College Dublin in 1980, 1982 and 1987 respectively. His research interests cover information management, in particular multimedia information and management of personal information from lifelogging. He is a coordinator of the annual TRECVid video benchmarking activity since 2001 and is a founding principal investigator in the CLARITY Centre for Sensor Web Technologies at Dublin City University. He has published over 220 research papers and is a member of the editorial boards for 5 journals. Alan is a member of the IEEE Computer Society. 